\title{
"It's the best job in the world. And you even get paid for it."
}

\section{Patrick Eichenberger*}

Prof. Dr. Patrick Eichenberger ist Dozent für Philosophie, Zeitgeschichte und Wirtschaftsethik an der HWZ, Hochschule Zürich für Wirtschaft und verbringt momentan ein Sabbaticaljahr in Port Macquarie, Australien.

Korrespondenz:

Prof. Dr. Patrick Eichenberger

23, Timber Ridge

Port Macquarie NSW 2444

Australia

patrick.eichenberger@fhhwz.ch
Auch heute kennt Australien einen erheblichen Mangel an medizinischer Versorgung, sobald die Ballungsgebiete verlassen werden, die weit weniger als $10 \%$ der australischen Gesamtfläche ausmachen. Australien zählt 24 Millionen Einwohner auf rund 7,8 Millionen Quadratkilometern. Das unglaubliche Abenteuer des «Royal Flying Doctor Service of Australia» ist eine Erfolgsmischung aus Medizin, Fliegerei und Funkverkehr, die von einem Pioniergeist seit der vorletzten Jahrhundertwende beseelt ist. Die Arbeit als fliegender - oftmals rettender - Outback-Arzt könnte auch für Schweizer Ärztinnen und Ärzte eine faszinierende Aufgabe sein. Alleine auf sich gestellt, fernab von Zivilisation, wo immer wieder neue Ausnahmesituationen ein gutes Urteilsvermögen, Selbstbewusstsein, Können, gesunden Menschenverstand und gelegentlich Improvisation verlangen. Wie begann der RFDS?

\section{Royal Flying Doctor Service of Australia - eine Organisation mit Idealismus}

Man muss versuchen, sich in die damalige Zeit zu versetzen, als im australischen Outback auf einer Fläche von $300000 \mathrm{~km}^{2}$ in Western Australia und weiteren 1,5 Millionen $\mathrm{km}^{2}$ in den Northern Territories nur mal gerade zwei Ärzte zur Verfügung standen!

1911 nahm der Geistliche John Flynn im Auftrag der presbyterianischen Kirche den Aufbau elementarer Buschspitäler und Übernachtungsmöglichkeiten im entfernten Outback in Angriff, um die Schrecken der Einsamkeit zu lindern (Missionsarbeit), aber auch um eine gewisse medizinische Grundversorgung zu ermöglichen.

Zahlreiche Beispiele aus der Zeit belegen, wie damals eine «world full of worries» das Outback dominierte. Das bekannteste Einzelschicksal, das in Australien seinerzeit dank dem Missionar Flynn die Runde machte, stammt aus dem Jahr 1917, als Jimmy Darcy, ein Viehzüchter (stockman), schwer erkrankte. Jimmy wurde von seinem Freund in einer zwölfstündigen (!) Holperfahrt 30 Meilen $(50 \mathrm{~km}$ ) weiter nach Halls Creek gebracht. Dort verfügte nur der Postbeamte

\section{Royal Flying Doctor Service of} Australia (RFDS): une possibilité d'enrichissement professionnel pour les médecins suisses?

Aujourd'hui encore, l'Australie connaît une forte pénurie de médecins dès que l'on s'éloigne des zones urbaines. Les agglomérations font beaucoup moins de $10 \%$ de la surface de l'ensemble du pays. L'Australie compte $\mathbf{2 4}$ millions d'habitants pour 7,8 millions de kilomètres carrés. La fascinante aventure de ce service de «médecins volants» est la réunion très réussie de la médecine, de l'avion et des liaisons radio: une entreprise qui est animée $d^{\prime}$ un esprit de pionnier et qui fonctionne depuis le début du siècle dernier. Le travail en tant que médecin aviateur - et souvent sauveur de vies humaines - pourrait se révéler une tâche passionnante pour des médecins suisses. Seuls, loin de la civilisation, les docteurs qui s'engagent ainsi sont en butte à des situations d'exception qui demandent un bon jugement, de la personnalité, du savoir, de bons rapports avec l'être humain et parfois aussi le goût de l'improvisation.

F. W. Tuckett über gewisse Kenntnisse in erster Hilfe. Sogleich erkannte Tuckett die Schwere in Jimmys Zustand. Auf der Suche nach ärztlicher Hilfe telegrafierte der Postbeamte nach Wyndham und Derby. Erfolglos. Schliesslich morste er verzweifelt seinem ehemaligen Erste-Hilfe-Ausbildner, Dr. Holland, ins 2000 Meilen (3300 km!) entfernte Perth. Symptombeschreibungen wurden nach Perth gemorst. Weitere Fragen wurden in «Piepstöne» gewandelt und die Antworten nach weiteren Minuten zurückübermittelt. Ent- 


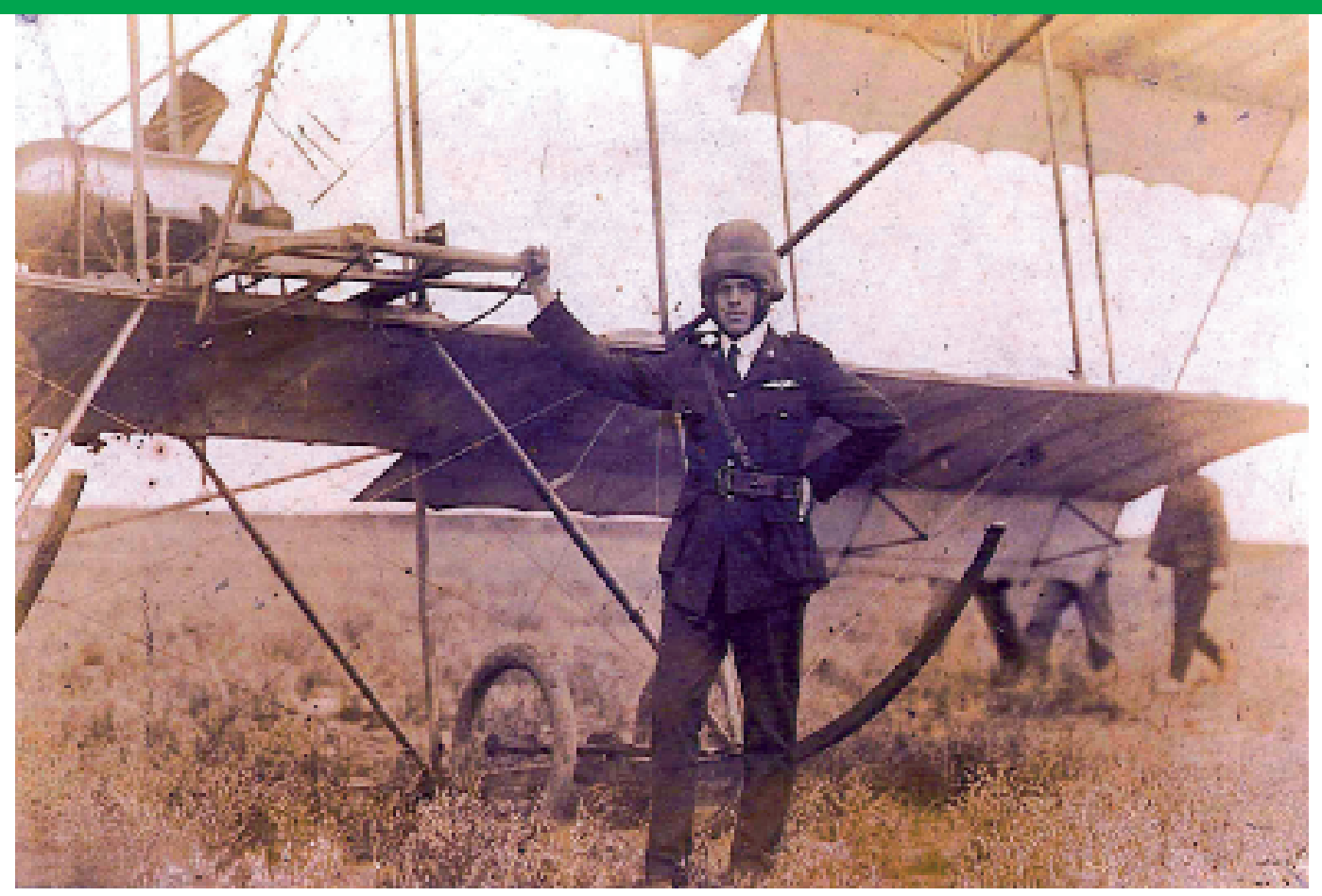

Abbildung 1

Das Fliegen im Blut: Lionel Cooke am Melbourne Cup 1915.

scheidende Morsesignale ... do-di-do-di-di-di-do ... Die Diagnose stand nach einer knappen halben Stunde Morsen fest. Holland übermittelte in der Folge die präzisen Anweisungen für die zweistufige Gallenblasenoperation, die Tuckett sofort mit einem Klappmesser in improvisierter Form durchführte. Beide Eingriffe dauerten lange und waren schmerzhaft. Danach machte sich Dr. Holland auf den langen Weg nach Halls Creek. In insgesamt zehn Tagen fuhr er von Perth in einem Schaftransportboot nach Halls Gap; weiter gings im Ford Modell «T»; dann auf dem Pferdewagen und das letzte Stück zu Fuss. Als Dr. Holland in Halls Creek ankam, war der Patient bereits gestorben. Obwohl beide zuvor über Fernanweisung (Morsen!) durchgeführten Gallenblaseneingriffe erfolgreich waren, war eine gleichzeitige Malariaerkrankung und Blinddarmentzündung des Patienten nicht erkannt worden. Daran war Jimmy gestorben.

Die tragische Geschichte von «Jimmy the stockman» hat die Gemüter damals in Australien genauso bewegt wie die zahllosen Zeitungsmeldungen vom Ersten Weltkrieg, der seinerzeit in Europa tobte. Eines war klar: der australische Outback brauchte Ärzte und Pflegepersonal!

Flynn, der Missionar, sah 1917 dank Leutnant Clifford Peel, einem Medizinstudenten aus Victoria mit einem Faible für die Fliegerei, die ideale Lösung: Moderne Flugapparate könnten grosse Distanzen relativ schnell überwinden, um die Behandlung von Patienten auch im entfernten Outback zu ermöglichen. Flynn war von dieser Idee besessen und veröffentlichte noch im gleichen Jahr im «Church's Inlander Magazine» diesen revolutionären Lösungsvorschlag. Peel konnte die Folgen seiner Idee nicht mehr erleben. Er wurde 1918 über der deutsch-französischen Front abgeschossen.

Nach zehnjähriger Geldsammlung konnte Flynn schliesslich 1928 den «Royal Flying Doctor Service of Australia» (RFDS) aus der Taufe heben. Heute betreibt die RFDS einen 24-Stunden-Service an 365 Tagen im Jahr im dünnbesiedelten Outback, aber auch in anderen Randregionen Australiens. Das Lieblingsflugzeug der RFDS ist heute die Pilatus PC-12 made in Switzerland!

\section{Ein Pionierarzt als Beispiel der RFDS}

Angesprochen auf seine siebenjährige Tätigkeit als «Flying Doctor» von 1968 bis 1974 sagte mir Dr. David Cooke als erstes: «You know Patrick, it's the best job in the world. And you even get paid for it!».

David stammt aus einer australischen Flugpionierfamilie. Schon bevor es überhaupt Flugzeuge in Australien gab, beschäftigte sich Urgrossvater William Cooke in Perth als Astronom mit den Sternen. Er gründete 1897 das erste Observatorium von Western Australia. Grossvater Lionel Cooke besass die RAF-Lizenz Nr. 11 und war Fluginstruktor im Ersten Weltkrieg (Abb. 1). Vater Rolla Cooke wurde als freiwilliger «Aussie»Pilot im Zweiten Weltkrieg an die RAF ausgeliehen und kam in einem Spitfire bei der Luftschlacht um England 1941 ums Leben. Er hatte insgesamt nur 220 Stunden Flugerfahrung! David, Jahrgang 1941, fliegt, seit er 17 Jahre alt 
ist. Er weist heute eine persönliche Logzeit von über 11000 Flugstunden auf! Sein Sohn ist Berufspilot im Chartergeschäft und macht weltweite Flugzeugüberführungen.

Als David 1968 zum «Royal Flying Doctor Service of Australia» kam, waren die diagnostischen Möglichkeiten im Flugzeug noch rudimentär. Aber genau das machte es besonders interessant. Zeitweise musste David bei 40 Grad Hitze auf dem Rollfeld unter dem Schatten der Tragfläche eine Notoperation mit unvollständigen oder ungenauen Diagnosedaten vornehmen. Für diesen Job sind keine Mediziner geeignet, die im Studium mit Höchstnoten brilliert haben, meint David. Besonnene Handwerkertypen mit einem guten Gespür sind hier gefragt, die pragmatisch und schnell handeln, gerade weil keine $« 2^{\text {nd }}$ opinion» eingeholt werden kann. Ab und zu musste sich David auch als Zahnarzt und Veterinär einbringen. Denn so schnell kommt kein Mediziner mehr ins Outback! Um ein guter, brauchbarer Flying Doctor zu werden, brauchte David geschätzte 18 Monate «on the job». Einerseits, um am Funk vorab die richtige Entscheidung zu fällen (go or no go) und um anderseits beim Patienten schnell die richtige Diagnose zu stellen.

\section{Ein Beispiel für eine falsche «GO-Entscheidung»}

Eines Morgens brüllt ein «Ringer» (= Stockman, Cattleman, Cowboy) ins Funkgerät:

Ringer: «Doc, my mate's crook. U got a come fast!» (crook = krank)

Dr. Cooke: «Can u describe anything specific. What happened?»

Ringer lauter: «Doc, I'm telling u. My mate's bloody crook!»

Dr. Cooke: «Just describe what happened and how he is?»

Ringer brüllt: «He's bloody dying.»

Dr. Cooke: «We'll be there in two hours!»

Dr. Cooke hatte sich für GO entschieden. Es war in seinem ersten Jahr als Flying Doctor. Als David nach 90 Minuten Flugzeit nahe der Farm landen konnte, stellte sich heraus, dass der Stockman nur erheblich über den Durst «gesoffen» hatte. Sylvesterkater. Er hätte es ahnen sollen: Es war an einem 1. Januar.

Zwei weitere Einsatzanekdoten zur Illustration:

\section{Beispiel aus 1971: Hirnerschütterung nach Rodeowettbewerb?}

Beim morgendlichen Funkverkehr wurde ein Notfall westlich von Cairns gemeldet. Ein Rodeoreiter sei beim Wettbewerb vom Pferd gestürzt und habe sich dabei womöglich eine Hirnerschütterung zugezogen. Schädelbasisbruch nicht ausgeschlossen. Patient liegt im Koma. Schon beim Endanflug auf die Naturpiste war die Sandwolke des nahenden Landrovers auszumachen. Als der Fahrer ausstieg, kam die Überraschung. Der Patient sei vor einer Stunde aus der Bewusstlosigkeit aufgewacht und habe darauf bestanden, auch beim zweiten Durchgang des Rodeoturniers weiterzumachen. Es sei unmöglich gewesen, den Kerl von seinem Vorhaben abzubringen. Bei der Konsultation nach dem Wettbewerb konnte David nur ein paar deftige Prellungen beim Reiter feststellen. «No worries, mate! Just a rough guy.»

\section{Beispiel 1972: Schwertfisch durchbohrt rechten Lungenflügel!}

Auf einer dem Great Barrier Reef vorgelagerten Insel nahe Lizard Island sei beim Fischen ein Marlin (Schwertfisch) aus dem Wasser gesprungen und habe dem armen Fischer den rechten Lungenflügel durchbohrt. Die rechte Lunge sei völlig dekomprimiert. Eine Landung nur auf dem benachbarten Lizard Island möglich. Also musste David ab der Landung zuerst mit einem Traktor zum Bootssteg fahren, um anschliessend im Schnellboot zum Verunfallten gefahren zu werden. Nachdem der Fischer transportfähig gemacht werden konnte, nun die ganze Strecke umgekehrt: Schnellboot, Traktor und der schwierige Rückflug, weil damals keine Druckkabinen im Flugzeug vorhanden waren. Es konnte nicht mehr als mit 50 bis 100 Fuss Höhe über Wasser zurückgeflogen werden, um die Lunge des Patienten nicht unnötig zu schädigen. Der Patient überlebte.

\section{Die Grenzen der Flying Doctors}

Die Grenzen der Flying Doctors kamen 1971 und 1974 besonders zum Ausdruck, als weite Teile von NW-Queensland von schlimmen Überschwemmungen heimgesucht wurden. Die meisten Pisten in NW-Queensland waren unter Wasser. Also wurde der Dienst provisorisch mit langsameren und teureren Hubschraubern aufrechterhalten. Der eine Hinflug zu einer Patientin dauerte wegen des starken Gegenwindes länger als geplant. Der Treibstoff (AVGAS) ging fast aus, als kurz vor Einbruch der Dunkelheit auf einer kleinen Anhöhe, umgeben von einem Meer von Wasser, im Gulf of Carpentaria gelandet werden konnte. Mit der Einsatzstelle Mt. Isa bestand kein Funkkontakt. Die Zeitung «Sydney Herald» hatte als Schlagzeile «Flying Doc missing in Gulf of Carpentaria» am Folgetag auf der Titelseite und schickte sofort ein Suchteam im Flugzeug 
nach Queensland. Als die Reporter den Hubschrauber entdeckten, funkten sie zum gestrandeten Team: «What do you need?» Die Antwort kam sofort: «80 liter AVGAS and 2 packs of cigarettes.» Der Titelseite des «Sydney Herald» am nächsten Morgen konnte «Flying doc's alive needs AVGAS and cigarettes» entnommen werden. Der Patientin konnte mit etwas Verspätung schliesslich doch noch geholfen werden.

Die teuren und gelegentlich misslungenen Hubschraubereinsätze zeigten die Grenzen des Flying Doctor Service auf: Australien war einfach

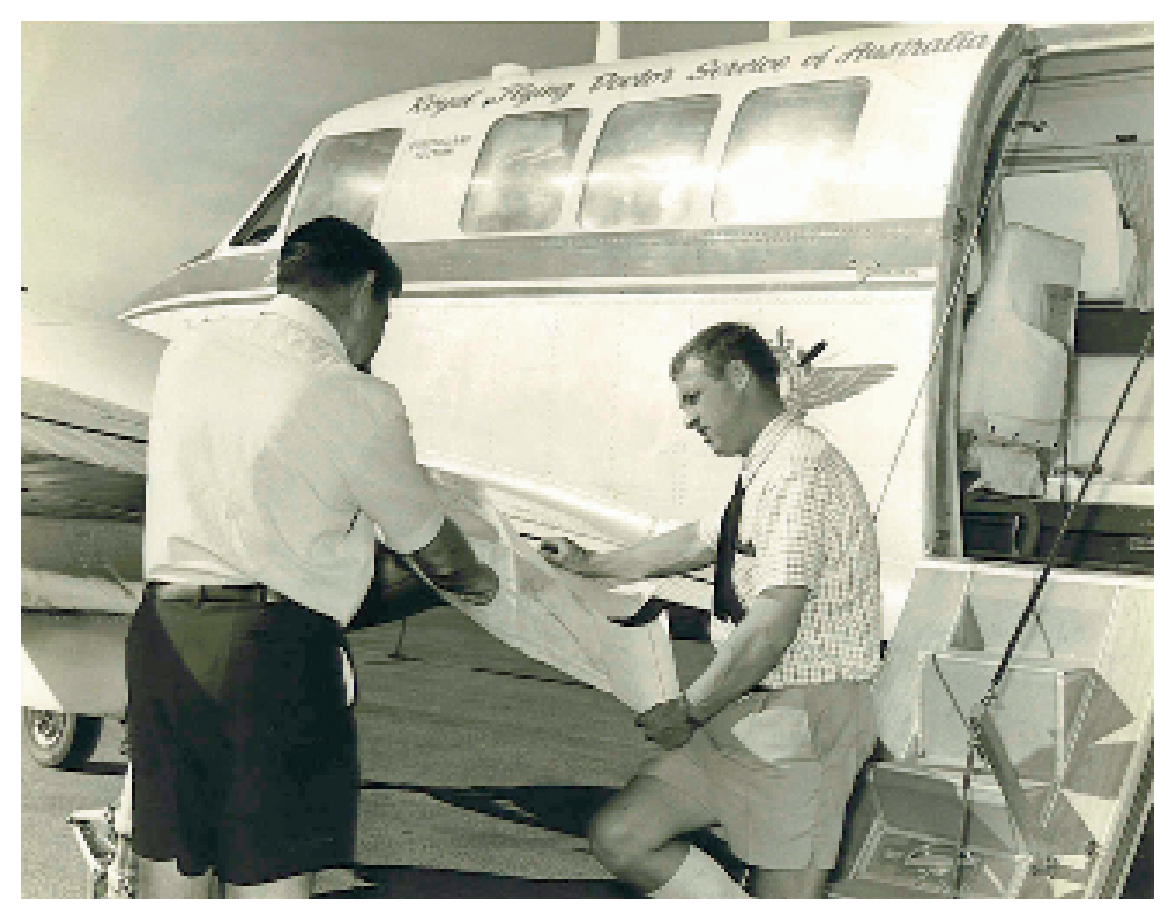

Abbildung 2

David Cooke als junger «flying doctor» im Jahr 1971 bei einer Einsatzbesprechung ...

zu gross! David machte den Vorschlag, für spezielle Notfälle (keine Landemöglichkeit etwa bei Überschwemmungen) mit Fallschirmabsprüngen rechtzeitig zu den Patienten vorzudringen. Tatsächlich absolvierte er 1971 zwei Absprünge samt Notarztausrüstung, um die Machbarkeit dieser Extremvariante $\mathrm{zu}$ demonstrieren. Allerdings musste bislang nie auf diesen Grenzfall zurückgegriffen werden.

Das Faszinierende an der Aufgabe als «Medical Officer» beim RFDS ist auch das wachsende Verständnis im Umgang mit einer Natur der Extreme, meint David. Eine Natur, die allzu häufig vom modernen Menschen nicht verstanden wird. Im Outback zeigt die Natur Mechanismen, die richtig gelesen werden müssen und einen zwingen, entsprechende Vorkehrungen zu treffen, die gelegentlich auch Improvisation bedingen.
Zwei kleine Geschichten, um dieses verbreitete Unverständnis im australischen Outback besser zu illustrieren.

1. Ausgerechnet bei einem Trainingsflug mit einem jungen Assistenzarzt musste wegen eines Motorschadens knapp 25 km vom Stützpunkt Mt. Isa (900 km SW von Cairns) in der Wüste an einem späten Januarvormittag notgelandet werden. Der Mayday-Funkspruch wurde quittiert. Hilfe sei in einer Stunde vor Ort. Als David und der junge Arzt beim Flugzeug auf die Nothilfe warteten, stellte David folgende Frage: «Was würdest Du jetzt und hier tun, wenn Du keinen Funkspruch hättest absetzen können und der Wasservorrat bei der Notlandung verlorengegangen wäre?» Nach ein paar Augenblicken antwortete der Trainee: «Kein Problem. Ich sehe den Funkturm von Mt. Isa schon von hier aus. Distanz $25 \mathrm{~km}$. Also gute 5 bis 6 Marschstunden. Ich nehme meinen Sonnenschutz und marschiere los. Gegen 18 Uhr spätestens bin ich auf dem Stützpunkt.» David schmunzelt und antwortet: «Du würdest dort nie ankommen. In einer Stunde ist die Tagestemperatur im Simpson Desert bei etwa 45 Grad. Der Wassergehalt in Deinem Blut nimmt ab (verdampft). Dein Blut verdickt. In zwei bis drei Stunden erleidest Du einen Hirnschlag oder Herzinfarkt und Du bist wenig später tot. Die Lösung: Du wartest unter der Tragfläche des Flugzeugs liegend ab, bis die Sonne untergeht. Erst dann marschierst Du los.»

2. Neulich, beim Durchqueren des Gibson Desert, hatte es ein Touristenpaar unterlassen, beim Abgangsort ihre Route samt Ankunftszeit am nächsten Ziel bekanntzugeben. Der Mietwagen erlitt eine Panne und die Fremden verfügten weder über ein Funkgerät noch hatten sie genügend Wasser dabei. Als ein Farmer zufällig zwei Tage später die gleiche Strecke befuhr, waren die beiden Touristen bereits verdurstet. Hätten sie daran gedacht, dass sich im Kühler über 12 Liter nutzbares Wasser befindet, wäre ein Überleben wahrscheinlich gewesen. In Australien wird kein Frostschutzmittel in das Kühlmedium geschüttet.

Noch heute ist Dr. med. David Cooke mit seinen 65 Jahren ein flugbegeisterter und aktiver Landarzt, der zweimal die Woche von Port Macquarie nach South West Rocks in seiner eigenen Beechcraft Bonanza fliegt, um dort seine zweite Praxis zu betreiben.

Gegenwärtig sind die Ziele für einen RFDSArzt (Medical Officer) in etwa gleich wie vor dreissig Jahren geblieben: «To provide primary 


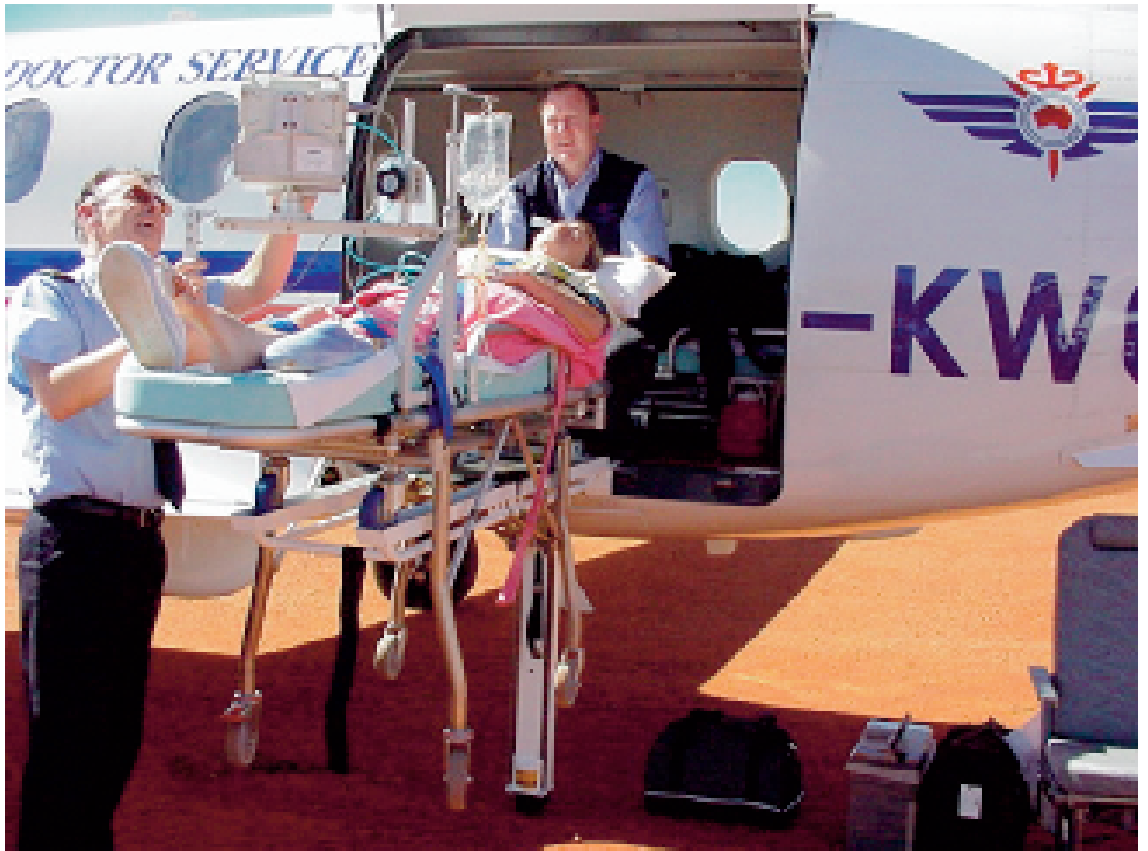

Abbildung 3

... und heute mit zeitgemässem Equipment.

health care, emergency retrieval and inter-hospital transfer services to people living and working in regional, rural and remote Queensland.»

Die Voraussetzungen für diese spannende Arbeit sind in zwei Bereiche zu trennen:

\section{Bewerbung als Arzt bei der RFDS}

- Abgeschlossenes Arztstudium (das mit dem Australischen Studium mindestens gleichwertig sein muss)

- 12monatige Verpflichtungszeit als Minimum an einem der Queensland-Stützpunkte Cairns (100000 Einwohner), Mt Isa (25 000), Rockhampton (65000), Charleville (3500) oder Kowanyama Aboriginal Community (1200, $735 \mathrm{~km}$ NW von Cairns)

- Englischkenntnisse: Bestehen des IELTS-Tests mit je Minimalnote 7 in den vier Testbereichen writing, listening, speaking, reading

- geeignete Persönlichkeit, um in einem multidisziplinären Team zu arbeiten

- Bereitschaft, ab einem isolierten und weitentfernten Standort zu arbeiten

- ein Kandidat sollte idealerweise Erfahrungen in folgenden Bereichen mitbringen:

- Allgemeinmedizin

- Notfallmedizin

- Anästhesie
- Geburtshilfe und Gynäkologie

- Flugmedizin sowie

- Sonderkenntnisse in Medizin in ländlichen und isolierten Gegenden.

- Nützlich und hilfreich sind absolvierte Lehrgänge in

- Prehospital trauma life support

- Advanced life support in Geburtshilfe

- Advanced paediatric life support

- Early Management of severe trauma and cross-cultural awareness.

- Arbeitsbeschreibung der RFDS

- eigenverantwortliche Arbeit mit minimaler Beaufsichtigung

- kollegiale Zusammenarbeit mit externen Leistungserbringern

- gute und wirksame Kommunikation sowie zwischenmenschliche Fähigkeiten

- Bereitschaft für flexible Arbeitszeiten

- hoher Reiseanteil, Flugstunden. Hohe Abwesenheitszeit vom Basisstützpunkt

- gültiger Fahrausweis (PW)

\section{Arbeitsrechtliche Voraussetzungen} die nach erfolgreicher Bewerbung beim RFDS gerne geschaffen werden (Australien braucht Mediziner!)

- Arbeitsvisum wird beantragt aufgrund eines Nachweises von Deckungsgleichheit zwischen Stellenbeschreibung und Kandidatenprofil (in der Regel durch den RFDS in der Provinz Queensland).

- Der Arzt muss in der zuständigen Provinz (wie kantonale Zulassung; hier in der Regel Queensland) beim zuständigen Medical Board akkreditiert werden (Qualifikation und Erfahrung).

- Der Kandidat kann beauftragt werden, ein Telefon- oder persönliches Interview zu bestehen.

- In gewissen Fällen können beobachtete, klinische Interviews angeordnet werden.

- Der Bewerber kann alternativ auch das Australian Medical Council Exam bestehen. Allerdings ist dieser Schritt aufwendiger und zeitlich langwieriger als das Erfüllen der oben aufgelisteten Voraussetzungen.

Eine gegenwärtig gültige Stellenbeschreibung (6 Seiten) als Arzt beim RFDS kann vom Autor gerne per E-Mail unter patrick.eichenberger@ fhhwz.ch verlangt werden. Quelle siehe www. flyingdoctor.net. 